\title{
Use of Point-of-Care Ultrasound for Evaluation of Extravascular and Intravascular Fluid Status in Pediatric Patients Maintained on Chronic Hemodialysis
}

\author{
Orly Haskin ${ }^{a, b}$ Yafa Falush ${ }^{a}$ Miriam Davidovits ${ }^{a, b}$ Hadas Alfandary ${ }^{a, b}$ \\ Shelly Levi ${ }^{a, b}$ Ron Berant ${ }^{b, c}$
}

anstitute of Nephrology, Schneider Children's Medical Center of Israel, Petach Tikva, Israel; bSackler Faculty of Medicine, Tel Aviv University, Tel Aviv, Israel; 'Department of Pediatric Emergency Medicine, Schneider Children's

Medical Center of Israel, Petach Tikva, Israel

\section{Keywords}

Bedside ultrasound · Fluid status · Hemodialysis · Pediatrics in B-lines number during dialysis $(r=0.39, p<0.01)$. Percent of blood volume drop correlated with post-dialysis IVC/aorta ratio $(r=0.48, p<0.001)$. A higher percent of symptomatic episodes occurred with post-dialysis IVC/aorta ratio $<0.8$ versus $\geq 0.8$ ( 39.1 vs. $15.2 \%, p=0.036$ ). Four patients were hypertensive, a clinical parameter implying fluid overload, in only one sonographic evaluation indicated fluid overload. Eight patients were clinically determined to be euvolemic, in three of them sonographic evaluation discovered covert fluids. Conclusion: Bedside ultrasound is a single modality that can be used to assess both extravascular and intravascular fluid status. It may contribute to clinical decisions differentiating fluid-related versus fluid-unrelated hypertension and identifying patients with covert fluids. @2021 The Author(s).

Published by S. Karger AG, Basel

\section{Introduction}

Determination of true dry weight (DW) in pediatric patients maintained on chronic hemodialysis is of outmost importance. Chronic fluid overload contributes to karger@karger.com www.karger.com/bpu

Karger $\stackrel{\text { ' }}{5}$

GOPEN ACCESS
(C) 2021 The Author(s)

Published by S. Karger AG, Basel

This is an Open Access article licensed under the Creative Commons Attribution-NonCommercial-4.0 International License (CC BY-NC) (http://www.karger.com/Services/OpenAccessLicense), applicable to the online version of the article only. Usage and distribution for commercial purposes requires written permission.
Correspondence to:

Orly Haskin, orly.haskin@gmail.com 
cardiovascular morbidity, while excess fluid removal can result in intradialytic hypotension and cardiac stunning [1-3]. Traditional clinical parameters for assessing DW rely on clinical examination for signs of excess fluid, as well as the presence of hypertension as a marker of fluid overload. On the other hand, the development of intradialytic symptoms, when probing for DW, helps determine the lowest achievable post-dialysis weight, prescribing it as DW. The assumption that blood pressure correlates directly with extracellular volume has been challenged, whereas the development of intradialytic symptoms during dialysis may indicate excess ultrafiltration rates without necessarily attaining DW [4]. Thus, more accurate methods for objective fluid assessment in hemodialysis patients are needed.

Point-of-care ultrasound (POCUS) has become more common and useful in assessing both extravascular and intravascular volume status in pediatric patients. Sonographic lung B-lines have been used as a measure of extravascular lung water [5-8]. B-lines are generally absent in euvolemic patients and appear before clinical symptoms or signs of fluid overload $[5,6,9]$. The number of $\mathrm{B}$-lines pre-dialysis has been shown to be associated with the accumulated weight and the reduction in B-lines during dialysis has been shown to be proportional to the amount of fluid removed $[4-6,10]$. The presence of $>5$ $\mathrm{B}$-lines on lung ultrasound is an early sign of pulmonary congestion [9].

Inferior vena cava (IVC) diameter and IVC collapsibility index (IVCCI) are sonographic parameters that change in accordance with intravascular volume status $[11,12]$. An IVCCI $>50 \%$ is predictive of low CVP [13]. For both of these parameters the need to correct for patient's body surface area and heart rate, as well as technical errors, preclude them from being easily used $[4,11,12$, 14]. The IVC/aorta ratio is a reliable sonographic parameter of dehydration in pediatric patients [13, 15-18]. The IVC/aorta ratio can be assessed rapidly at bedside with good reliability [17]. In euvolemic healthy children, mean IVC/aorta ratio is $1.01 \pm 0.15$ [18]. An IVC/aorta ratio $<0.8$ was found to be a marker of severe volume depletion $[13,16,17]$.

The aim of our study was to compare the clinical assessment of fluid status to sonographic parameters of fluid status in pediatric patients undergoing chronic hemodialysis. Our study is the first to use a single bedside modality to examine both extravascular and intravascular volume and the first to examine the IVC/aorta ratio in hemodialysis patients.

\section{Materials and Methods}

This was a single-center prospective observational study. The study group included all pediatric patients treated with chronic hemodialysis at Schneider Children's Medical Center of Israel during October 2016 to December 2017. The study was approved by the Institutional Research Review Committee. Written informed consent and assent were obtained from patients and their parents before participation.

\section{Clinical Evaluation}

Patients were evaluated before and after dialysis during 6 consecutive dialysis sessions for pre- and post-dialysis weight, systolic blood pressure (SBP), and diastolic blood pressure (DBP). Symptoms and physical signs of fluid overload before dialysis, such as edema and lung crackles and symptoms of excess fluid removal, such as abdominal pain, cramping, headache, hypotension, and tachycardia, during dialysis, were recorded. During dialysis, patients were monitored with a blood volume sensor, and the percent of blood volume drop (BVD \%) at the end of dialysis was recorded. Intradialytic weight gain was calculated based on the difference between the current pre-dialysis weight minus the previous postdialysis weight. SBP and DBP indices were calculated according to the formula: $(\mathrm{SBP}$ index $=[$ measured SBP $] /[95$ th percentile SBP for sex and height]; DBP index = [measured DBP] $/[95$ th percentile DBP for sex and height]). Assessment of the patients' fluid status (euvolemic, fluid overload, or low-volume status) was carried out routinely at the end of each week by the attending nephrologist. This was done using clinical parameters including pre- and postdialysis weight, blood pressure, intradialytic weight gain, and BVD $\%$. Decisions regarding DW and antihypertensive treatment were made by the attending nephrologist who was blinded to the sonographic findings.

Data collected from patients' files included baseline renal disease, age and dialysis vintage, residual urine output, the use of antihypertensive medications, and echocardiography result. Laboratory measurements included monthly single-pool KT/V or standard weekly $\mathrm{KT} / \mathrm{V}$ and patients' hemoglobin level at the time of the study.

\section{Sonographic Evaluation}

During the 6 dialysis sessions, sonographic parameters were assessed in addition to clinical parameters, both pre- and postdialysis. Sonography was performed using Zonare $\mathrm{Z} 1$ pro device with a micro-convex $\mathrm{C} 10-3$ probe. All ultrasound examinations were done by a single physician, a graduate of a POCUS fellowship and experienced in POCUS, with over 1,000 scans. Post-dialysis evaluation was done within $30 \mathrm{~min}$ of completing dialysis. The ultrasound examination was taken with the patient in a semi-recumbent position. Total numbers of lung B-lines were measured and counted in 28 views over the lungs, as previously reported [5]. For patients under $20 \mathrm{~kg}, 14$ position assessment was used (parasternal, midclavicular, and anterior axillary/midaxillary sites) [6]. The IVCCI was defined as the percent of decrease in IVC diameter in inspiration versus expiration

\section{IVC diameter on expiration - IVC diameter on inspiration IVC diameter on expiration}

Measurement of IVC diameter for the IVCCI was done with the patient supine during normal expiration and inspiration; the transducer was placed just inferior to the xiphoid process in a longitudi- 


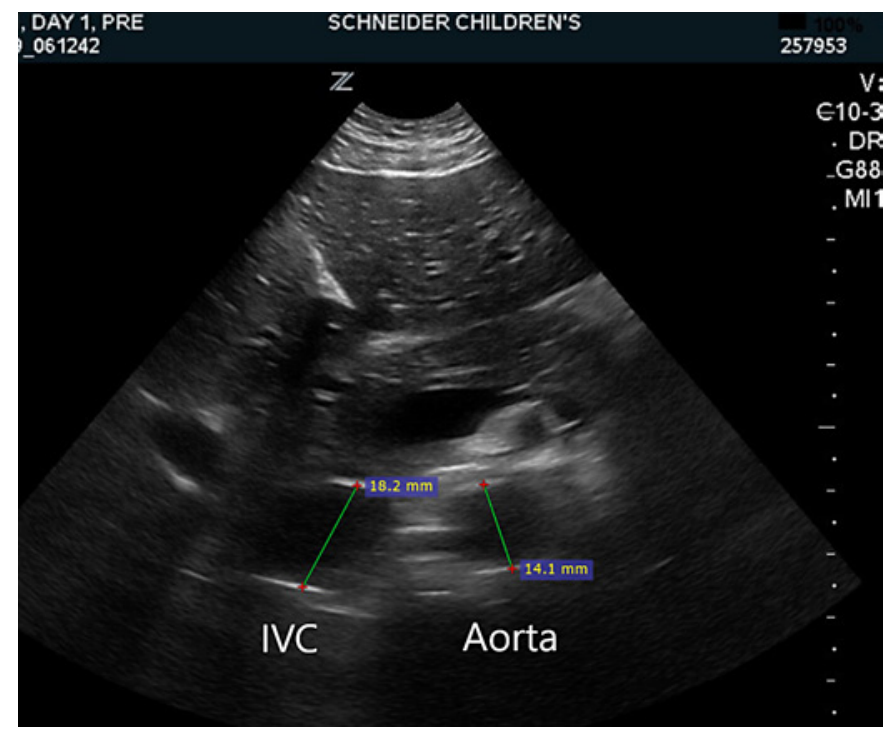

Fig. 1. Measurement of the IVC/aorta ratio. The ultrasound probe is placed in the transverse plane, just inferior to the xiphoid process, where both the IVC and the aorta can be seen and measured. IVC, inferior vena cava.

nal direction and slid to the right until the IVC was demonstrated. The anteroposterior IVC diameter was measured during expiration and inspiration $[11,14]$. The IVC/aorta ratio was calculated as the IVC diameter/aorta diameter measured with the probe placed just inferior to the xiphoid process in a transverse plane $[16,17]$ (shown in Fig. 1). Patients were classified according to post-dialysis sonographic parameters as: fluid overloaded ( $>5$ B-lines post-dialysis); low-volume status ( $\leq 5 \mathrm{~B}$-lines and IVC/aorta $\leq 0.8$ post-dialysis); and euvolemic ( $\leq 5 \mathrm{~B}$-lines and IVC/aorta $>0.8$ post-dialysis).

\section{Statistical Analysis}

Statistical analysis was performed using SAS software versus 9.4. Descriptive statistics are presented as means and standard deviations. Comparisons between continuous variables were done using the Student's $t$ test. Comparisons between categorical variables were done using Fisher's exact test. Pearson correlation was used to measure the strength of linear association between 2 variables. A $p$ value of $<0.05$ was considered statistically significant.

\section{Results}

Thirteen patients were included in the study group. One patient with severe developmental delay was excluded because of difficulty in obtaining images due to lack of co-operation. The demographic characteristics of $12 \mathrm{pa}$ tients are described in Table 1. Ten patients were dialyzed 3 times/week; 2 patients with significant residual renal function; and normal blood pressure were dialyzed only 2 times/week, without ultrafiltration. Dialysis sessions
Table 1. Demographic and clinical characteristics of 12 pediatric hemodialysis patients

Baseline characteristics
Age, years (mean $\pm \mathrm{SD}$; range)

Dialysis vintage, years (median, IQR)

Baseline disease, $n$

CAKUT

Hereditary

Glomerulonephritis

Unknown

Residual renal function

(urine output $>500 \mathrm{~mL} / \mathrm{d}), n(\%)$

Antihypertensive medications, $n$ (\%)

Echocardiography findings

Normal, $n(\%)$

$\mathrm{LVH}, n(\%)$

Decreased ejection fraction, $n(\%)$

Hemoglobin levels, mg/dL (mean \pm SD)
$14.3 \pm 4.9(2.3-20)$

$1.05(0.7,1.5)$

5

3

3

1

$9(75)$

$2(17)$

$1(8.3)$

$10.95 \pm 0.97$
SD, standard deviation; IQR, interquartile range; CAKUT, Congenital anomalies of the kidney and urinary tract; $\mathrm{LVH}$, left ventricular hypertrophy.

were 3-5 h long depending on patient's volume status and the need for ultrafiltration. All patients had single-pool $\mathrm{KT} / \mathrm{V}$ of at least 1.4. The 2 patients who were dialyzed only twice a week had a standard KT/V $>2$ (including dialysis and patient's residual renal function).

Pre-dialysis versus post-dialysis clinical parameters and sonographic measurements are presented in Table 2. All clinical parameters (weight, $\mathrm{SBPi}$, and DPBi) as well as sonographic parameters (total B-lines number, IVC/aorta ratio, and max IVC diameter) were significantly lower post-dialysis than pre-dialysis. \%IVCCI was significantly higher post-dialysis versus pre-dialysis. Ultrafiltration volume and weight change, during dialysis correlated positively with change in B-lines number pre-dialysis versus post-dialysis $(r=0.39, p=0.0008 ; r=0.34, p=0.004$, respectively). BVD \% correlated positively with post-dialysis IVC/aorta ratio $r=0.48, p<0.001$ and negatively with reduction in $\mathrm{B}$-lines number $r=-0.39, p=0.0014$ (less negative BVD correlates with a higher post-dialysis IVC/ aorta ratio and a lower reduction in B-lines number).

In 16 (22\%) of 72 dialysis sessions, patients had symptoms of excess fluid removal. In all these sessions, ultrafiltration was stopped, though dialysis continued. In 4 sessions, fluids were given. The proportion of symptomatic episodes with post-IVC/aorta ratio $<0.8$ was higher than the proportion with a ratio $\geq 0.8$ (39.1 vs. $15.2 \%, p=$ 0.036 ). Similarly, the proportion of symptomatic epi- 
Table 2. Clinical and sonographic parameters pre- and post-dialysis

\begin{tabular}{lllll}
\hline & Pre-dialysis & Post-dialysis & $p$ value & $95 \%$ CI \\
\hline Weight (mean \pm SD), kg & $41.5 \pm 12.8$ & $40.2 \pm 12.3$ & $<0.0001$ & $1.04-1.44$ \\
SBPi (mean \pm SD) & $0.96 \pm 0.12$ & $0.94 \pm 0.13$ & 0.0066 & $0.007-0.04$ \\
DBPi (mean \pm SD) & $0.97 \pm 0.17$ & $0.94 \pm 0.19$ & 0.016 & $0.005-0.055$ \\
Total B-lines (mean \pm SD), $n$ & $7.69 \pm 7.46$ & $4.49 \pm 5.05$ & $<0.0001$ & $2.14-4.25$ \\
\%IVCCI (mean \pm SD) & $24.6 \% \pm 10.9$ & $34.8 \% \pm 12$ & $<0.0001$ & $2.17-3.76$ \\
IVC/aorta ratio (mean \pm SD) & $1.1 \pm 0.2$ & $0.9 \pm 0.2$ & $<0.0001$ & $2.17-3.76$ \\
Max IVC diameter (mean \pm SD), mm & $15.3 \pm 3.8$ & $12.3 \pm 4.1$ & $<0.0001$ & $2.2-3.8$
\end{tabular}

$\mathrm{SD}$, standard deviation; SBPi, systolic blood pressure index; DBPi, diastolic blood pressure index; \%IVCCI, inferior vena cava collapsibility index; IVC, inferior vena cava; Max, maximal.

Table 3. Mean values of clinical and sonographic parameters of the 6 dialysis sessions for each patient

\begin{tabular}{|c|c|c|c|c|c|c|c|c|c|c|}
\hline \multirow{2}{*}{ Patient } & \multirow{2}{*}{$\begin{array}{l}\text { mean } \\
\text { IDWG, kg }\end{array}$} & \multirow{2}{*}{$\begin{array}{l}\text { post-dialysis } \\
\text { WT }>\text { DW, } \\
\mathrm{kg}\end{array}$} & \multirow{2}{*}{ HTN } & \multirow[t]{2}{*}{ BVD $\%$} & \multirow{2}{*}{$\begin{array}{l}\text { Fluid status per } \\
\text { clinical assessment }\end{array}$} & \multicolumn{2}{|c|}{ pre-dialysis } & \multicolumn{2}{|c|}{ post-dialysis } & \multirow{2}{*}{$\begin{array}{l}\text { Fluid status per } \\
\text { sonographic } \\
\text { parameters }\end{array}$} \\
\hline & & & & & & $\begin{array}{l}\text { mean } \\
\text { B-lines, } n\end{array}$ & $\begin{array}{l}\text { mean } \\
\text { IVC/aorta }\end{array}$ & $\begin{array}{l}\text { mean } \\
\text { B-lines, } n\end{array}$ & $\begin{array}{l}\text { mean } \\
\text { IVC/aorta }\end{array}$ & \\
\hline 1 & 0.42 & 1.32 & Yes & -6.1 & Fluid overload & 8.5 & 1.04 & 7.3 & 1 & Fluid overload \\
\hline 2 & 2.2 & 0.87 & Yes & -11.7 & Fluid overload & 1.2 & 1.19 & 0.17 & 0.78 & Low-volume status \\
\hline 5 & 1.79 & 0.28 & No & -14.8 & Euvolemic & 22.7 & 1.28 & 14.8 & 0.99 & Fluid overload \\
\hline 6 & 1.7 & 0.08 & No & -11.2 & Euvolemic & 14.8 & 1.17 & 7.7 & 1.23 & Fluid overload \\
\hline 7 & 0 & 0.16 & No & -5.4 & Euvolemic & 8 & 1.2 & 6.33 & 1.03 & Fluid overload \\
\hline 8 & 2.08 & 0.47 & No & -16.8 & Euvolemic & 3 & 0.97 & 1.33 & 0.6 & Low-volume status \\
\hline 9 & 1.35 & 1.58 & No & -12.9 & Fluid overload & 4.3 & 0.97 & 1.5 & 0.89 & Euvolemic \\
\hline
\end{tabular}

IDWG, intradialytic weight gain; WT, weight; DW, dry weight; HTN, hypertension; BVD\%, percent of blood volume decrease; IVC, inferior vena cava.

sodes with post-IVCCI $\geq 50 \%$ was higher than the proportion with post-IVCCI $<50 \%$ (55.5 vs. $18.3 \%, p=0.026$ ).

\section{Clinical Versus Sonographic Parameters of Fluid Status}

Table 3 shows mean values of clinical and sonographic parameters of the 6 dialysis sessions for each patient with determination of fluid status based on clinical assessment versus sonographic parameters. Except for hypertension, none of the patients had clinical symptoms or physical signs of fluid overload. Four patients (1-4) were hypertensive pre- and post-dialysis with $\mathrm{SBPi} \geq 1$ (Table 3 ). Patient 1 had on average $7 \mathrm{~B}$-lines post-dialysis as well as post-dialysis IVC/aorta ratio of 1 , indicating fluid overload implying hypertension is related to fluids. Clinically, he was not reaching DW with low BVD \% also indicating fluid overload. Patients 2 and 3 had low pre- and post-dialysis B-lines number and low post-dialysis IVC/ aorta ratio, indicating low-volume status implying hypertension is not related to fluids. Clinically, patient 2 was having recurrent symptoms during dialysis concurring with low-volume status. Patient's 4 sonographic parameters were discrepant (high mean post-dialysis B-lines number, but low post-dialysis IVC/aorta ratio). This patient was classified as fluid overload per clinical assessment; however, he was having repeated symptoms during dialysis concurring with low-volume status and the low post-dialysis IVC/aorta ratio. The relatively high post-di- 
alysis B-lines number may be explained by the patient's interstitial lung disease.

All other patients (except patient 9) were clinically determined as euvolemic as they were not hypertensive and were reaching their DW. Of them, 3 patients $(5,6$, and 7$)$ were classified as fluid overload by sonography since they had on average $>5 \mathrm{~B}$-lines at the end of dialysis indicating covert fluid. Patients with post-dialysis B-lines $>5$ also had a significantly higher post-dialysis IVC/aorta ratio $1.0 \pm 0.2$ versus $0.81 \pm 0.22 p<0.001$ than patients with $<5$ B-lines. Patient 9 was not reaching DW, so was determined to be fluid overload by clinical assessment; however, sonographic assessment found him to be euvolemic. Overall, only in 4 patients, there was an agreement between clinical and sonographic assessment.

\section{Discussion}

The determination of true DW in hemodialysis patients continues to be a clinical challenge. Traditional methods that rely on blood pressure measurements and probing for DW are inaccurate. More objective methods are needed to assess patients' fluid status. POCUS is increasingly being utilized in a variety of clinical settings to improve the assessment of patients' fluid status [5-10,13, 15-17].

We showed that all sonographic parameters significantly changed during dialysis (Table 2), indicating these measurements are influenced by fluid removal during dialysis. The change in B-lines number correlated positively with UF volume and weight change during dialysis as shown in the previous studies $[6,10,19]$. Post-dialysis IVC/aorta ratio correlated positively with a lower percent of BVD at the end of dialysis as both are markers of intravascular volume status.

Our study is the first to examine the IVC/aorta ratio as a marker of intravascular volume status in dialysis patients. We found that a higher percent of symptomatic episodes occurred with a post-dialysis IVC/aorta $<0.8$ and post-dialysis IVCCI $>50 \%$. This is in agreement with previous studies in children with severe dehydration unrelated to dialysis $[13,16,17]$. The fact that in only $39.1 \%$ of sessions with symptoms post-dialysis IVC/aorta ratio was $<0.8$ can be explained by the timing of the measurements. Since the measurements were performed only after disconnecting the patients from dialysis, it allowed time for refilling between symptoms onset and sonographic assessment.

Analyzing sonographic parameters in patients with hypertension, we were able to show that POCUS may help us differentiate between volume-dependent and volume-independent hypertension. As seen in 4 hypertensive patients, in 1 patient hypertension was likely related to fluids given at the high post-dialysis B-lines number and IVC/aorta ratio, while in 3, hypertension was likely unrelated to fluids, given the low IVC/aorta ratio. Torterue et al. [20] similarly showed that IVC diameter measurement may help differentiate between volume dependent to volume independent hypertension. IVC diameter, however, is not a reliable marker to assess for fluid overload in pediatric hemodialysis patients $[10,20]$.

Lung B-lines have been used as a measure of extravascular volume in multiple studies [5-8]. They are superior to echocardiography and bioimpedance spectroscopy in detecting volume overload in dialysis patients $[10,21,22]$. In accordance with previous studies, we also demonstrated that sonographic assessment may aid in discovering covert fluids in patients who are normotensive $[5,10]$. Five of our patients repeatedly had $>5$ B-lines at the end of dialysis, as well as a relatively high IVC/aorta ratio, indicating fluid overload, only 2 of them were hypertensive. In none of them, DW was challenged. Subclinical volume overload was found to be an independent predictor of death and cardiac events in adult hemodialysis patients $[22,23]$. It was recently shown that lung-ultrasoundguided strategy for DW reduction can reduce ambulatory blood pressure levels in dialysis patients [24]. Using POCUS as a screening method to detect covert fluids may help improve patients' outcome.

Our study is the first to use a single modality to assess for both extravascular and intravascular fluid status. This allows for a relatively short, easy-to-use bedside technique by a single operator. Previous studies that looked at combined modalities of lung ultrasound, bioimpedance spectroscopy, and echocardiogram are more cumbersome and require higher operating skills [10, 21, 22]. Sonographic evaluations in our study were done in a separate room inside the dialysis unit with an average examination time of 15-20 min. Previous studies showed that 10-20 sonographic examinations under direct observation are required in order to obtain good proficiency in lung B-lines and IVC/aorta ratio measurements $[17,25]$.

It is important to remember that relaying only on lung B-lines for assessment of fluid status may be misleading, as B-lines may also be present in patients with intercurrent illness and parenchymal lung disease [26, 27]. In our study, 1 patient showed discrepancy in sonographic parameters with a high post-dialysis B-line number but a low post-dialysis IVC/aorta ratio. This patient had interstitial lung disease due to ANCA vasculitis so B-lines may not 
solely indicate fluid overload. Measuring both B-lines number and IVC/aorta ratio add credibility to sonographic results when both parameters support the same conclusion regarding fluid status. Overall, in our study, in 8 of 12 patients, there was a disagreement between clinical assessments of fluid status to sonographic assessment.

Our study has several limitations. First, it had the small sample size and the repeated measures in each patient, which may have skewed our statistical results. Second is the observational nature of our study, which did not assess intervention based on our sonographic findings. We also realized that repeat sonographic assessment at each dialysis session may not be practical in real life. However, the use of bedside ultrasound as a monthly screening method for covert fluid, and its use in hypertensive patients to asses for fluid-related hypertension may be feasible.

\section{Conclusion}

We have shown that bedside sonographic assessment of pediatric hemodialysis patients for both extravascular and intravascular volume, may add valuable information regarding fluid status that cannot be assessed based on clinical parameters alone. The increasing availability of POCUS and its ease of use warrant further investigations, as to whether it can improve clinical management. Further prospective interventional studies are needed to determine whether ultrasound-based dry weight assessment will improve cardiovascular morbidity in pediatric hemodialysis patients.

\section{Statement of Ethics}

Ethical review board: The Ethics (Helsinki) Committee at the Rabin Medical Center (Reference number 202822) approved the study protocol. Study ID number: 0448-16-RMC. Written informed consent and assent were obtained from patients and their parents before participation.

\section{Conflict of Interest Statement}

The authors have no conflicts of interest to declare.

\section{Funding Sources}

Funding was not required for this study.

\section{Author Contributions}

Orly Haskin MD, participated in design, acquisition, analysis, and drafting of the work. Yafa Falush participated in design and acquisition of the work. Miriam Davidovits MD, participated in the analysis and critical revising of the work. Hadas Alfandary MD participated in acquisition, analysis, and critical revising of the work. Shelly Levi MD, participated in acquisition, analysis, and critical revising of the work. Ron Berant MD, participated in design, acquisition, analysis, and drafting of the work.

\section{References}

1 Paglialonga F, Consolo S, Galli MA, Testa S, Edefonti A. Interdialytic weight gain in oligoanuric children and adolescents on chronic hemodialysis. Pediatr Nephrol. 2015;30:9991005.

2 Chazot C, Wabel P, Chamney P, Moissl U, Wieskotten S, Wizemann V. Importance of normohydration for the long-term survival of haemodialysis patients. Nephrol Dial Transplant. 2012;27:2404-10.

3 Geer JJ, Shah S, Williams E, Arikan AA, Srivaths $P$. Faster rate of blood volume change in pediatric hemodialysis patients impairs cardiac index. Pediatr Nephrol. 2017;32:341-5.

4 Rosner MH, Ronco C. Techniques for the assessment of volume status in patients with end stage renal disease. Semin Dial. 2014; 27(6):538-41.

5 Noble VE, Murray AF, Capp R, Sylvia-Reardon M, Steele DJR, Liteplo A. Ultrasound assessment for extravascular lung volume in pa- tients undergoing hemodialysis. Time course for resolution. Chest. 2009;135:1433-9.

6 Allinovi M, Saleem M, Romagnani P, Nazerian P, Hayes W. Lung ultrasound: a novel technique for detecting fluid overload in children on dialysis. Nephrol Dial Transplant. 2017;32:541-7.

7 Kaskinen AK, Martelius L, Kirjavainen T, RautiainenAndersson PS, Andersson S, Pitkänen $\mathrm{OM}$. Assessment of extravascular lung water by ultrasound after congenital cardiac surgery. Pediatr Pulmonol. 2017;52(3):345-52.

8 Al Deeb M, Barbic S, Featherstone R, Dankoff J, Barbic D. Point of care ultrasonography for the diagnosis of acute cardiogenic pulmonary edema in patients presenting with acute dyspnea: a systematic review and meta-analysis. Acad Emerg Med. 2014;21(8):843-52.

9 Picano E, Pellikka PA. Ultrasound of extravascular lung water: a new standard for pulmonary congestion. Eur Heart J. 2016;37(27): 2097-104.
10 Allinovi M, Saleem MA, Burgess O, Armstrong C, Hayes W. Finding covert fluid: methods for detecting volume overload in children on dialysis. Pediatr Nephrol. 2016; 31:2327-35.

11 Mandelbaum A, Ritz E. Vena cava diameter measurement for estimation of dry weight in haemodialysis patients. Nephrol Dial Transplant. 1996;11(Suppl 2):24-7.

12 Krause I, Birk E, Davidovits M, Cleper R, Blieden L, Pinhas $\mathrm{L}$, et al. Inferior vena cava diameter: a useful method for estimation of fluid status in children on haemodialysis. Nephrol Dial Transplant. 2001;16: 1203-6.

13 Babaie S, Behzad A, Mohammadpour M, Reisi $\mathrm{M}$. A comparison between the bedside sonographic measurements of the inferior vena cava indices and the central venous pressure while assessing the decreased intravascular volume in children. Adv Biomed Res. 2018;7:97-111. 
14 Kutty S, Li L, Hasan R, Peng Q, Rangamani S, Danford DA. Systemic venous diameters, collapsibility indices, and right atrial measurements in normal pediatric subjects. J Am Soc Echocardiogr. 2014;27:155-62.

15 Kosiak W, Swieton D, Piskunowic M. Sonographic inferior vena cava/aorta diameter index, a new approach to the body fluid status assessment in children and young adults in emergency ultrasound-preliminary study. Am J Emerg Med. 2008;26:320-5.

16 Chen L, Hsiao A, Langhan M, Riera A, Santucci KA. Use of bedside ultrasound to assess degree of dehydration in children with gastroenteritis. Acad Emerg Med. 2010;17:1042-7.

17 Levine AC, Shah SP, Umulisa I, Munyaneza RB, Dushimiyimana JM, Stegmann K, et al. Ultrasound assessment of severe dehydration in children with diarrhea and vomiting. Acad Emerg Med. 2010;17:1035-41.

18 Chen L, Kim Y, Santucci KA. Use of ultrasound measurement of the inferior vena cava diameter as an objective tool in the assessment of children with clinical dehydration. Acad Emerg Med. 2007;14:841-5.
19 Fu Q, Chen Z, Fan J, Ling C, Wang X, Liu X, et al. Lung ultrasound methods for assessing fluid volume change and monitoring dry weight in pediatric hemodialysis patients. $\mathrm{Pe}$ diatric Nephrol. 2021;36(4):969-76.

20 Torterue X, Dehoux L, Macher MA, Niel O, Kwon T, Deschenes G, et al. Fluid status evaluation by inferior vena cava diameter and bioimpedance spectroscopy in pediatric chronic hemodialysis. BMC Nephrology. 2017;18: 373-80.

21 Alexiadis G, Panagoutsos S, Roumeliotis S, Stibiris I, Markos A, Kantartzi K, et al. Comparison of multiple fluid status assessment methods in patients on chronic hemodialysis. Int Urol Nephrol. 2017;49:525-32.

22 Siriopol D, Hogas S, Voroneanu L, Onofriescu M, Apetrii M, Oleniuc M, et al. Predicting mortality in haemodialysis patients: a comparison between lung ultrasonography, bioimpedance data and echocardiography parameters. Nephrol Dial Transplant. 2013;28: 2851-9.
23 Zoccali C, Torino C, Tripepi R, Tripepi G, D’Arrigo G, Postorino M, et al. Pulmonary congestion predicts cardiac events and mortality in ESRD. J Am Soc Nephrol. 2013;24: 639-46.

24 Loutradis C, Sarafidis PA, Ekart R, Papadopoulos C, Sachpekidis V, Alexandrou ME, et al. The effect of dry weight reduction guided by lung ultrasound on ambulatory blood pressure in hemodialysis patients: a randomized controlled trial. Kidney Int. 2019;95: 1505-13.

25 Bedetti G, Gargani L, Corbisiero A, Frassi F, Poggianti E, Mottola G. Evaluation of ultrasound lung comets by hand-held echocardiography. Cardiovasc Ultrasound. 2006;4: 34.

26 Caiulo VA, Gargani L, Caiulo S, Fisicaro A, Moramarco E, Latini G, et al. Lung ultrasound in bronchilolitis: comparison with chest Xray. Eur J Pediatr. 2011;170:1427-33.

27 Martelius L, Heldt H, Lauerma K. B-lines on pediatric lung sonography: comparison with computed tomography. J Ultrasound Med. 2016;35:153-7. 